\title{
An E.S.R. study of the triplet diamions of 1,3,5-triphenylbenzene and 2,4,6-triphenyl-sym-triazine
}

\author{
by J. A. M. VAN BROEKHOVEN $\dagger$, J. L. SOMMERDIJK $\ddagger$ \\ and E. DE BOER \\ Department of Physical Chemistry, University of Nijmegen, Nijmegen, \\ The Netherlands
}

(Received 4 December 1970)

Upon prolonged alkali reduction in ethereal solvents 1,3,5-triphenylbenzene (Tpb), 2,4,6-triphenyl-sym-triazine ( $\mathrm{Tpt}$ ) and 2,4,6-tritolyl-sym-triazine ( $\mathrm{Ttt}$ can be reduced to their dinegative ions. Dissolved in rigid solvent matrices these ions produce E.S.R. spectra which are characteristic for randomly oriented triplet systems. Hückel and SCF MO calculations indicate that these dianions have a twofold degenerate lowest anti-bonding level.

Although the ions $\mathrm{Tpb}^{2-}$ and $\mathrm{Tpt}^{2-}$ are iso-electronic their magnetic properties prove to be strikingly different. While $\mathrm{Tpb}^{2-}$ has a triplet ground state irrespective of solvent, counter ion and temperature, $\mathrm{Tpt}^{2-}$ (and also $\mathrm{Ttt}^{2-}$ ) has a singlet ground state with a thermally accessible triplet state in solvents with poorly solvating properties and has a triplet ground state in better solvating media. Furthermore, the zero field splitting parameters $D$ and $E$ of the lowest triplet states of $\mathrm{Tpt}^{2-}$ and $\mathrm{Ttt}^{2-}$ are markedly different from those obtained from $\mathrm{Tpb}^{2-}$. The former show a considerably larger $D$ value than $\mathrm{Tpb}^{2-}$ and, in contrast to $\mathrm{Tpb}^{2-}$, the ions $\mathrm{Tpt}^{2-}$ and $\mathrm{Ttt}^{2-}$ have a non-zero $E$ value in poorly solvating solvents, pointing to a non-trigonal spin distribution of the triplet systems.

An explanation of the observed differences is given in terms of current MO theories. The results suggest that in the case of $\mathrm{Tpt}^{2-}$ and $\mathrm{Ttt}^{2-}$ the association with the counter ions even in strongly solvating solvents is very strong.

\section{INTRODUCTION}

According to experiments by Jesse et al. [1], 1,3,5-triphenylbenzene (Tpb) and decacyclene can be reduced with an alkali metal in an ethereal solvent to their dinegative ions. These ions were found to have a triplet ground state and could therefore be studied with E.S.R. spectroscopy. Subsequently E.S.R. experiments proved that this was also the case for several other aromatic hydrocarbons dianions having trigonal symmetry, e.g. triphenylene ${ }^{2-}$, coronene $^{2-}$ and 2,4,6-triphenylsym-triazine ( $\mathrm{Tpt}^{2-}$, figure 1) [2-4]. This can be understood theoretically, since

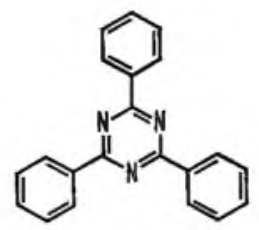

Figure 1. Structure diagram of 2,4,6-triphenyl-sym-triazine.

† Koninklijke/Shell Laboratory, Amsterdam.

‡ Philips Research Laboratories, Eindhoven. 
according to Hückel and self-consistent field (SCF) molecular orbital (MO) calculations these systems have a twofold degenerate lowest anti-bonding level. By taking into account electron correlation it can be shown that the two additional $\pi$-electrons yield a triplet ground state.

The foregoing holds true only if the dianions are not perturbed by the counter ions. Experimentally this situation may be realized to a good approximation in solvents with high solvating power, i.e. glymes which have the general formula $\mathrm{CH}_{3}\left[\mathrm{OCH}_{2} \mathrm{CH}_{2}\right]_{n} \mathrm{OCH}_{3}$.

In solvents with less solvating power, e.g. 2-methyltetrahydrofuran (MTHF), these dianions are strongly associated with the alkali counter ions, resulting in the formation of ion pairs or ion triples [2-8]. This association manifests itself by a drastic change of the zero-field-splitting (ZFS) parameters as compared with those of the unperturbed dianions. In the case of triphenylene ${ }^{2-}$ and coronene ${ }^{2-}$ this results in a non-zero $E$ value indicating a non-trigonal symmetry of the triplet system $[2,6]$. Apparently the cation(s) possesses a non-centric position with respect to the dianions. For triphenylene ${ }^{2-}$ the triplet state remains the lowest state under all circumstances [8], but for coronene ${ }^{2-}$ this is not the case. It shows a siuflet ground state in MTHF but a smoty

In this paper a systematic study is presented of the counter ion effects on the triplet E.S.R. spectra of $\mathrm{Tpb}^{2-}$, $\mathrm{Tpt}^{2-}$ and $\mathrm{Ttt}^{2-}$. From the temperature dependence of the magnetic susceptibility it was found that $\mathrm{Tpb}^{2-}$ has a triplet ground state irrespective of solvent or counter ion. This was explained by assuming that the counter ions in the ion pairs or ion triples possess such positions that they do not disturb the trigonal symmetry of the triplet spin distribution. Confirmation of this was obtained by comparison of the experimental with the calculated ZFS parameters $[7,8]$.

For $\mathrm{Tpt}^{2-}$ and $\mathrm{Ttt}^{2-}$, on the other hand, the multiplicity of the ground state was proved to be strongly dependent on solvent. In contrast to earlier observations [9] we found triplet spectra for the dianions of Tpt and Ttt. However, the multiplicity of the ground state was strongly dependent on the solvating power of the solvent. Also the ZFS parameters are considerably different from those obtained for $\mathrm{Tpb}^{2-}$. Comparison with Hückel and SCF MO calculations indicates a strong association between the dianions and the counter ions not only in apolar solvents like MTHF but also in the strongly solvating glymes.

\section{EXPERIMENTAL}

The E.S.R. experiments have been performed on a Varian $4502 \mathrm{X}$ band spectrometer equipped with a variable temperature control accessory and a field dial. The magnetic field was measured with an AEG gaussmeter and the frequency was monitored with a Hewlett-Packard $5245 \mathrm{~L}$ frequency counter. Variable temperatures between -160 and $-260^{\circ} \mathrm{C}$ were obtained by passing cold helium gas along the sample. The temperature was measured with a copper-constantan couple calibrated at liquid nitrogen and liquid helium temperature.

The dianions have been prepared in a standard way on a vacuum line. The solvents MTHF and 1,2-dimethoxyethane were dried over a sodium dispersion in paraffin, distilled in vacuo and stored over a $\mathrm{Na} / \mathrm{K}$ alloy. The polyethers dyglyme, triglyme and tetraglyme were dried on $\mathrm{Na} / \mathrm{K}$ alloy and distilled just before use. 


\section{EXPERIMENTAL RESULTS}

(a) Triphenylbenzene dianion $\left(T p b^{2-}\right)$

Prolonged reduction of $\mathrm{Tpb}$ with an alkali metal in an ethereal solvent results in the formation of the dinegative ion $[1,5]$. In a glassy matrix at $-160^{\circ} \mathrm{C} \mathrm{E.S.R}$. spectra could be recorded which are characteristic of a species with an electronic triplet state. Resonance signals have been detected both at half field $\left(\Delta M_{s}=2\right)$ and full field $\left(\Delta M_{s}=1\right)$ (see figure 2). In a preliminary communication [5] we reported the observation of three different classes of triplet species in solvents of varying composition. The same phenomena had previously been observed for the dianion of triphenylene [2]. Since then more detailed information has become available by varying more widely the composition of the triplet solutions. In table 1 a survey is

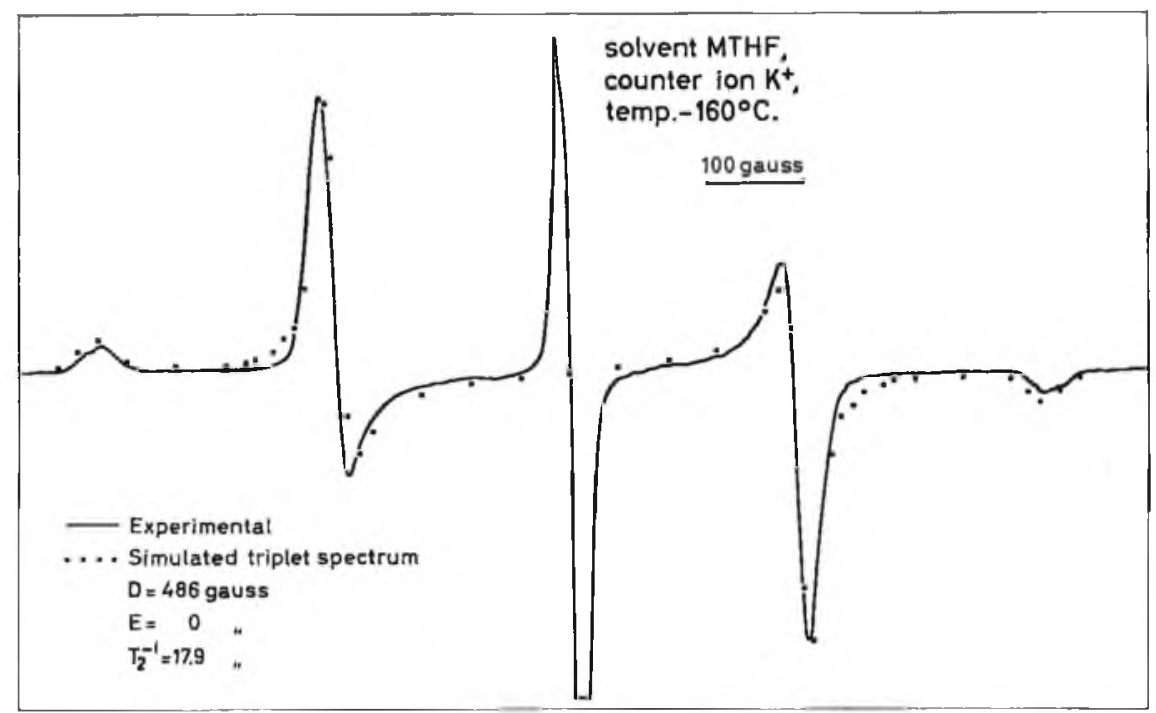

Figure 2. Experimental and calculated triplet spectrum of $\mathrm{Tpb}^{2-} 2 \mathrm{~K}^{+}$in $\mathrm{MTHF}$. The central line is due to the monoanion. For the calculation of the theoretical spectrum the equations given in ref. [24] were used.

presented of all experiments carried out on the dianions of Tpb. In pure MTHF only one triplet species was observed, except for $\mathrm{Tpb}$ reduced with $\mathrm{Li}$. The other experiments were carried out by successive addition of glymes to MTHF. In table 1 are indicated the various $D$ values of the distinct triplet species observed under these conditions. In all cases studied the intensity of the triplet E.S.R. signal varied linearly with the reciprocal of the temperature. The spectra are characterized by only one ZFS parameter $D$ ( $E$ being equal to zero).

It is clear from table 1 that in most cases successive introduction of small amounts of polyethers to $\mathrm{MTHF}$ solutions of $\mathrm{Tpb}^{2-}$ yield in principle three distinct triplet species differing largely in their ZFS parameters. It is also evident from the table that the smallest ZFS observed $(\approx 355$ gauss) is practically independent of the solvent and counter ion. This was also found for the triphenylene dianion [2]. $\mathrm{Li}$ reduced $\mathrm{Tpb}$ is somewhat exceptional when compared to the other alkali salts. Reduction of $\mathrm{Tpb}$ with $\mathrm{Li}$ in MTHF produced two triplet spectra superimposed with $D$ values of 473 and 347 gauss, respectively, the spectrum with the smaller $D$ 


\begin{tabular}{|c|c|c|}
\hline Counter ion & solvent & $D$ (gauss) \\
\hline $\mathrm{Li}$ & MTHF & $\begin{array}{l}473 \\
347\end{array}$ \\
\hline $\mathrm{Li}$ & M'THF/tetraglyme & $\begin{array}{l}473 \\
347\end{array}$ \\
\hline $\mathrm{Na}$ & MTHF & 523 \\
\hline $\mathrm{Na}$ & MTHF/DME & $\begin{array}{l}523 \\
432 \\
355\end{array}$ \\
\hline $\mathbf{N a}$ & MTHF/diglyme & $\begin{array}{l}523 \\
440 \\
361\end{array}$ \\
\hline $\mathrm{Na}$ & MTHF/triglyme & $\begin{array}{l}523 \\
440 \\
355\end{array}$ \\
\hline $\mathrm{Na}$ & MTHF/tetraglyme & $\begin{array}{l}523 \\
435 \\
360\end{array}$ \\
\hline $\begin{array}{l}\mathbf{K} \\
\mathbf{K}\end{array}$ & $\begin{array}{l}\text { MTHF } \\
\text { MTHF/DME }\end{array}$ & $\begin{array}{l}498,488 \dagger \\
498-458 \ddagger\end{array}$ \\
\hline $\mathrm{K}$ & MTHF/diglyme & $\begin{array}{l}498,488 \uparrow \\
405 \\
354\end{array}$ \\
\hline $\mathbf{K}$ & Diglyme & 354 \\
\hline $\mathbf{K}$ & MTHF/triglyme & $\begin{array}{l}498-455 \ddagger \\
405 \\
354\end{array}$ \\
\hline $\mathbf{K}$ & MTHF/tetraglyme & $\begin{array}{l}498,488,463 \uparrow \\
408 \\
354\end{array}$ \\
\hline $\mathbf{R b}$ & MTHF & 478 \\
\hline $\mathrm{Rb}$ & MTHF/triglyme & $\begin{array}{l}478 \\
400\end{array}$ \\
\hline
\end{tabular}

† Different triplet species can be observed with slightly different $D$ values (see ref. [5], figure $2 a$ ).

$\ddagger$ The $D$ value decreases continuously on addition of glyme.

Table 1. The $D$ value of the distinct triplet species of $\mathbf{T p b}^{2-}$ in MTHF and MTHFpolyglyme mixtures at $-160^{\circ} \mathrm{C}$.

value being predominantly present. When tetraglyme was added to this solution, the species with $D=473$ gauss was completely converted into the dianion with a $D$ value of 347 gauss. In the case of $\mathrm{Rb}$ reduced $\mathrm{Tpb}$ the triplet spectrum with the lowest $D$ value could not be obtained. Introduction of diglyme or triglyme resulted ultimately in a triplet spectrum with a $D$ value of 400 gauss.

(b) Triphenyltriazine $(T p t)$ and tritolyltriazine $(T t)$ dianions

Both Tpt which is isolectronic with triphenylbenzene and Ttt can be reduced to paramagnetic dianions. In pure MTHF a triplet spectrum could only be obtained with $\mathrm{Na}$ as reducing agent. The $\mathrm{ZFS}$ parameters characterizing the triplet spectrum are: $D=680$ gauss and $E=25$ gauss (figure 3 ). When polyethers are used as solvents Tpt and Ttt can be reduced by more alkali metals to their dinegative ions 


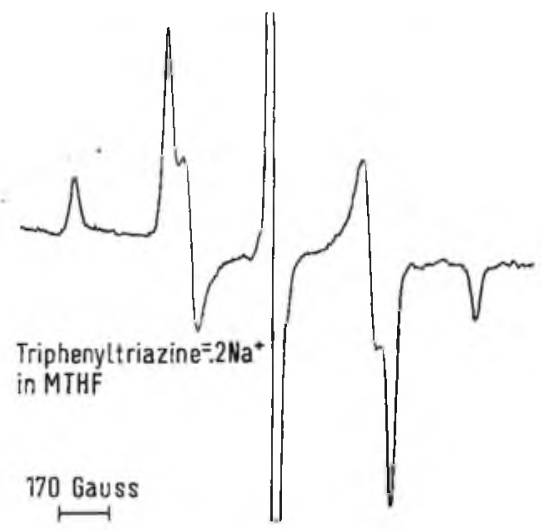

Figure 3. Triplet spectrum of $\mathrm{Tpt}^{2-2 \mathrm{Na}^{+}}$in $\mathrm{MTHF}$ at $-160^{\circ} \mathrm{C}$. The central line is due to the monoanion.

(table 2). Addition of tetraglyme to $\mathrm{Ttt}^{2-}$ dissolved in MTHF yielded three different triplet spectra; the $E$ value of the second spectrum could not be determined due to overlap with the other triplet spectra. Axial spectra, i.e. spectra characterized by only one ZFS parameter $D$, could be observed with $\mathrm{Li}, \mathrm{Na}, \mathrm{K}$ and $\mathrm{Rb}$ as reducing agent in pure diglyme or in a mixture of MTHF and polyethers. It can be seen from table 2 that the axial spectra are still somewhat dependent on solvent and counter ion, the $D$ value varying from 600 to 625 gauss.

The signal intensities of the axial triplet spectra varied linearly with the reciprocal of the temperature. The intensity of the non-axial spectra, however, showed a marked decrease when the temperature was lowered from $-160^{\circ} \mathrm{C}$ to $-250^{\circ} \mathrm{C}$. This was observed both for $\mathrm{Tpt}^{2-}$ and $\mathrm{Ttt}^{2-}$ in $\mathrm{MTHF}$, as is shown in figure 4. At the same time the signal intensities of the mononegative ions always

\begin{tabular}{|c|c|c|c|}
\hline Counter ion & Solvent & $D$ (gauss) & $E$ (gauss) \\
\hline$T p t^{2-L i}$ & Diglyme & 600 & 0 \\
\hline $\mathrm{Na}$ & MTHF & 680 & 25 \\
\hline $\mathrm{Na}$ & Diglyme & 616 & 0 \\
\hline $\mathrm{Na}$ & MTHF/tetraglyme & 613 & 0 \\
\hline $\mathrm{K}$ & Diglyme & 600 & 0 \\
\hline $\mathrm{Rb}$ & Diglyme & 611 & 0 \\
\hline $\mathrm{Ttt}^{2-} \mathrm{Li}$ & Diglyme & 600 & 0 \\
\hline $\mathrm{Na}$ & MTHF & 680 & 25 \\
\hline \multirow[t]{2}{*}{$\mathrm{Na}$} & MTHF/DME $(2: 1)$ & 680 & 25 \\
\hline & & 650 & ? \\
\hline $\mathrm{Na}$ & Diglyme & 625 & $\mathbf{0}$ \\
\hline \multirow{3}{*}{$\mathrm{Na}$} & MTHF/tetraglyme & 680 & 25 \\
\hline & & 650 & $?$ \\
\hline & & 625 & 0 \\
\hline $\mathbf{K}$ & Diglyme & 606 & 0 \\
\hline $\mathbf{R b}$ & Diglyme & 609 & 0 \\
\hline
\end{tabular}

Table 2. ZFS parameters of $\mathrm{Tpt}^{2-}$ and $\mathrm{Ttt}^{2-}$ in $\mathrm{MTHF}$, diglyme and $\mathrm{M} \mathrm{T}^{\prime} \mathrm{HF}$-polyglyme mixtures at $-160^{\circ} \mathrm{K}$. 
present together with the dianions, varied linearly with the reciprocal of the temperature.

\section{Discussion}

\section{(a) Triphenylbenzene dianion}

The dianions of $\mathrm{Tpb}$ and triphenylene have a feature in common. For both cases three distinct triplet species can be observed, which are ascribed to unperturbed dianions, ion pairs and ion triples $[2,5]$. As Tpb can be reduced to its dianion by more alkali metals than triphenylene, more can be said about the solvent and counter ion effect on the $D$ values of $\mathrm{Tpb}^{2-}$. The spectra with the lowest $D$ values must be ascribed to dianions which are not perturbed by cations. Usually one characterizes such ions as free ions or as very loosely bound ion pairs (triples). A distinction between these two possible structures cannot be inferred from E.S.R. experiments.

The $D$ value of the second class of triplet dianions increases with decreasing ionic radius of the counter ion; for $\mathrm{Li}, \mathrm{Na}, \mathrm{K}$ and $\mathrm{Rb}$ the $D$ values are 473,440 , 405 and 400 gauss, respectively. These triplet spectra are ascribed to ion pairs $\mathrm{Tpb}^{2-} \mathrm{Me}^{+}[2,8]$.

The $D$ values of the third class also increase with decreasing ionic radius of the cation, the $D$ values being 523,498 and 478 gauss for $\mathrm{Na}, \mathrm{K}$ and $\mathrm{Rb}$, respectively. These spectra are ascribed to ion triples $\mathrm{Tpb}^{2-} 2 \mathrm{Me}^{+}[2,8]$. The corresponding spectrum of $\mathrm{Tpb}^{2-} 2 \mathrm{Li}^{+}$was never observed: in most solvents the $\mathrm{Li}$ salts are completely solvated.

Two general features are evident from the spectra:

(a) the larger the ionic radius of the metal ion, the larger the amount of polyether necessary to generate successive triplet species like $\mathrm{Tpb}^{2-} \mathrm{Me}^{+}$ and non-associated $\mathrm{Tpb}^{2-}$.

(b) polyethers with more coordination sites available solvate more rapidly: $\mathrm{DME}<$ diglyme $<$ triglyme $<$ tetraglyme.

These features have also been observed for solvated mononegative ions [10]. The stronger solvation of the smaller ions is caused by the enhanced electrostatic interaction between the cations and the solvent molecules. The larger ions have a more diffuse charge distribution, which weakens the charge-dipole interaction. Polyethers with many coordination sites (oxygen atoms) available can form suitable solvent cages for the cations so that one large polyether molecule can completely solvate a metal ion.

On the base of quantitative measurements on $\mathrm{Tpb}^{2-}-2 \mathrm{~K}^{+}$(see ref. [2]) it may be concluded that the first two glyme molecules solvate the ion pair complex on the outside without changing the ion pair structure too abruptly. The third molecule solvates one cation completely to give the species $\mathrm{Tpb}^{2-} \mathrm{K}^{+}$, while the fourth glyme molecule solvates the second $\mathrm{K}^{+}$ion completely. Quantum-mechanical calculations of the ZFS parameters and of the term schemes of the various species proved to be in good agreement with the above explanation, if the cations in $\mathrm{Tpb}^{2-} \mathrm{Me}^{+}$and $\mathrm{Tpb}^{2-} 2 \mathrm{Me}^{+}$are located near the trigonal axis of $\mathrm{Tpb}^{2-}[7]$.

\section{(b) Triphenyltriazine dianion}

(i) The ground state of $T p t^{2-}$

Though the dianion of $\mathrm{Tpt}$ is isoelectronic with that of $\mathrm{Tpb}$ it behaves quite differently. The $D$ values of $\mathrm{Tpt}^{2-}$ are much larger than values measured for 


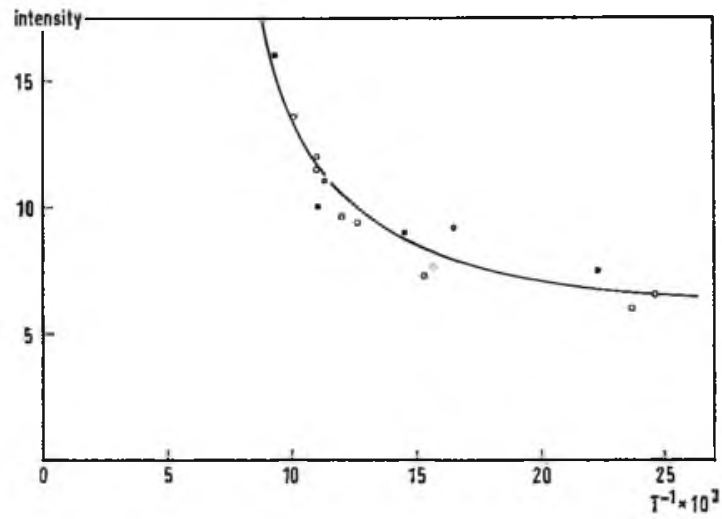

Figure 4. Intensity of the $\Delta m_{s}=2$ signal of $\mathrm{Tpt}^{2-}(\mathrm{O})$ and $\mathrm{Ttt}^{2-}(-)$ measured in MTHF as a function of the temperature.

$\mathrm{Tpb}^{2-}$ and the loss of the trigonal spin distribution of $\mathrm{Tpt}^{2-}$ in MTHF has not been observed in the case of $\mathrm{Tpb}^{2-}$. Addition of tetraglyme restored the threefold symmetry as has also been found for the triplet dianions of coronene [6] and triphenylene [5]. The axial triplet spectra of $\mathrm{Tpt}^{2-}$ (and $\mathrm{Ttt}^{2-}$ ) are generated by ground state triplet molecules because the triplet signal intensity follows Curie's law of the magnetic susceptibility. The intensity of the non-axial spectra, however, varied in a way characteristic for thermally excited triplets (figure 4). This means that the dianions producing these spectra have a singlet ground state with a nearby triplet state that can be thermally populated. From a qualitative term scheme
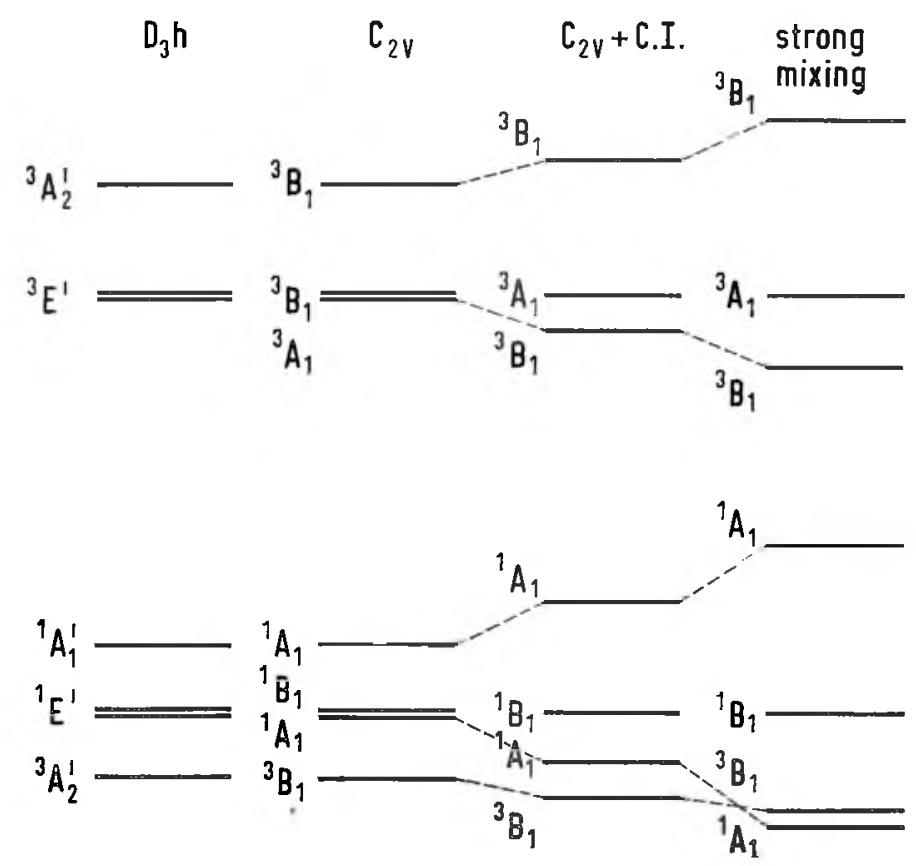

Figure 5. Qualitative term scheme for $\mathrm{Tpt}^{2-}$ under $D_{3 h}$ and $C_{2 v}$ symmetry. 
(figure 5) one sees that the strength of the non-symmetrical perturbation will determine whether the electronic ground state will be a ${ }^{1} A_{1}$ or a ${ }^{3} B_{1}$ state.

The magnetic susceptibility due to the thermally excited triplets will be determined both by the Boltzmann distribution between the Zeeman levels and between the orbital states ${ }^{1} A_{1}$ and ${ }^{3} B_{1}$ (figure 6 ). Under the condition that the zero-field splittings $X, Y$ and $Z \ll J$ the following expression can be derived for the magnetic susceptibility [11]

$$
\chi=\frac{2 g^{2} \beta^{2} N}{3 k} \cdot \frac{1}{T\left(1+\frac{1}{3} \exp (J / k T)\right)},
$$

where $N$ is Avogadro's number and the other symbols have their usual meaning.

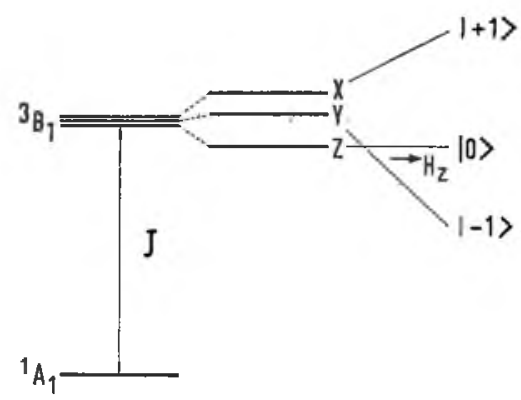

Figure 6. Lowest orbital states of $\mathrm{Tpt}^{2-}\left(\mathrm{Ttt}^{2-}\right) . \quad X, Y$ and $Z$ are zero-field energies of the ${ }^{3} B_{1}$ state. The Zeeman levels are denoted by $|+1\rangle,|0\rangle$ and $|-1\rangle$.

With this formula the singlet-triplet separation $J$ of $\mathrm{Tpt}^{2-} 2 \mathrm{Na}^{+}$can be calculated since the E.S.R. signal intensity is proportional to the magnetic susceptibility. It was found that $J$ varies with temperature, decreasing from $150 \mathrm{~cm}^{-1}$ at $100 \mathrm{~K}$ to $100 \mathrm{~cm}^{-1}$ at $30 \mathrm{~K}$. Because $J$ is much larger than the ZFS parameters $\left(\approx 0.05 \mathrm{~cm}^{-1}\right)$ the above made assumption $(J \gg X, Y, Z)$ proves to be valid. The variation of $J$ with temperature we ascribe tentatively to a decreased cation-anion interaction going to lower temperatures.

(ii) Calculation of the ZFS parameter $D$ and the singlet-triplet separation $J$

Hückel and SCF MO theory has been employed to calculate the ZFS parameters and the energy interval between the lowest singlet and triplet states of $\mathrm{Tpt}^{2-}$. In the Hückel approximation the parameters $\alpha_{\mathbf{X}}$ and $\beta_{\mathrm{CX}}$ (X being the hetero atom) are usually taken as [12]

$$
\begin{aligned}
\alpha_{\mathrm{X}} & =\alpha_{\mathrm{C}}+k \beta_{\mathrm{CC}}, \\
\beta_{\mathrm{CX}} & =h \beta_{\mathrm{CC}},
\end{aligned}
$$

where $h$ and $k$ are empirical parameters. Streitwieser suggested for the case of a nitrogen atom contributing one electron to the $\pi$ system the following values [12]

$$
\begin{aligned}
\alpha_{\mathrm{N}} & =\alpha_{\mathrm{C}}+0.5 \beta_{\mathrm{CC}}, \\
\beta_{\mathrm{CN}} & =\beta_{\mathrm{CC}} .
\end{aligned}
$$


In the Goeppert-Mayer and Sklar approximation [13]

$$
\alpha_{\mathbf{N}}=\alpha_{\mathbf{C}}-\left(W_{\mathbf{N}}-W_{\mathrm{C}}\right),
$$

where $W_{\mathrm{N}}-W_{\mathrm{C}}$ is a measure for the difference in attraction for an electron in a nitrogen core compared to a carbon core.

The value of $W_{\mathrm{N}}-W_{\mathrm{C}}$ and that of $\beta_{\mathrm{CN}}$ is taken from Anno [14]

$$
\begin{aligned}
\alpha_{\mathrm{N}} & =\alpha_{\mathrm{C}}-2.460 \mathrm{eV}, \\
\beta_{\mathrm{CN}} & =-2.576 \mathrm{eV} .
\end{aligned}
$$

With these two sets of different parameters Hückel and SCF calculations have been performed on $\mathrm{Tpt}^{2-}$.

The repulsion integrals, necessary for the SCF calculations, have been taken from Pariser and Parr $[15,16]$.

The energy scheme of the $\pi$ electronic levels is nearly the same as for $\mathrm{Tpb}^{2-}[7]$. The ZFS parameters have been calculated with the semi-empirical method of Van der Waals and Ter Maten [17] which was also used by Sommerdijk et al. [4, 7] to calculate the ZFS parameters of $\mathrm{Tp}^{2-}$ and $\mathrm{Tpb}^{2-}$. For the $\mathrm{C}-\mathrm{C}$ dipolar integrals the semi-empirical values given by these authors [17] were used. The $\mathrm{C}-\mathrm{N}$ and $\mathrm{N}-\mathrm{N}$ dipolar integrals have been derived from the corresponding integrals given by Capello and Pullman [18] properly corrected for $\sigma-\pi$ interaction.

\begin{tabular}{clcc}
\hline Parameters & Method & $D$ (gauss) & $E$ (gauss) \\
\hline$\left.\alpha_{\mathrm{N}}=\alpha_{\mathrm{C}}+0 \cdot 5 \beta_{\mathrm{CC}}\right)$ & Hückel & 520 & 0 \\
$\beta_{\mathrm{CN}}=\beta_{\mathrm{CC}}$ & SCF $+\mathrm{CI} \dagger$ & 330 & 0 \\
& & & \\
$\left.\alpha_{\mathrm{N}}=\alpha \mathrm{C}-2 \cdot 46 \mathrm{eV}\right)$ & Hückel & 490 & 0 \\
$\left.\beta_{\mathrm{CN}}=-2 \cdot 576 \mathrm{eV}\right)$ & SCF $+\mathrm{CI} \dagger$ & 290 & 0 \\
Experiment & & 600 & 0 \\
\hline
\end{tabular}

† Configurations up to $20 \mathrm{eV}$ from the ground configuration are included.

Table 3. Experimental and calculated ZFS parameters of $\mathrm{Tpt}^{2-}$.

The results of our calculations are given in table 3 together with the experimental value obtained in diglyme $(D=600$ gauss, $E=0)$. Because the fourth calculation is the most reliable, one is forced to conclude that there is a large discrepancy with experiment. Similar large deviations from experimental values were also found by Glasbeek [6] who calculated the ZFS parameters of coronene dianion. He suggested that the alkali ions would still be associated with the dianions even in polar solvents. We may have a similar situation here for $\mathrm{Tpt}^{2-}$ because the spectra obtained in polyethers still show some dependence on solvent and counter ion, as can be seen from table 2 .

To study this situation theoretically an MO calculation has been performed for $\mathrm{Tpt}^{2-} 2 \mathrm{Me}^{+}$with the metal ions on the trigonal axis ( $E$ must be zero) at $2 \AA$ distance from the aromatic plane. For the nitrogen the parameters given by Anno [14] were used. 
The perturbation of the cations was accounted for by using an extension of the method of McClelland [19] and of Reddoch [20]. In this method the cations are represented as point charges. The elements of the Hückel matrix are functions of the positions of the counter ions and are approximated as follows:

$$
\begin{aligned}
& \mathscr{H}_{i i}=\alpha+\left[\left(5 \cdot 6 / r_{i} M_{1}\right)+\left(5 \cdot 6 / r_{i} M_{2}\right)\right] \beta, \\
& \mathscr{H}_{i j}=\beta\left\{1+\left[2 \cdot 8 / r_{i} M_{1}+r_{j} M_{1}\right]+\left[2 \cdot 8 /\left(r_{i} M_{2}+r_{j} M_{2}\right)\right]\right\},
\end{aligned}
$$

in which $r_{i} M_{1}$ is the distance in Angströms of the cation $M_{1}$ to the carbon (or nitrogen) atom $i$.

Calculating the $D$ value from the modified Hückel MO's we found a value of 520 gauss. Constructing the SCF MO's on the basis of the modified Hückel MO's and applying first-order configuration interaction (CI) resulted in a lower $D$ value: $D=490$ gauss. This is much closer to the experimental value than the value calculated for the free dianion (see table 3). This may serve as an indication that even in the highly polar glymes the ions $\mathrm{Tpt}^{2-}$ and $\mathrm{Ttt}^{2-}$ are strongly associated with the counter ions.

In order to account for the non-zero $E$ value (25 gauss) obtained for $\mathrm{Tpt}^{2-}$ in MTHF a calculation was performed in which the cations were located in nontrigonal positions with respect to $\mathrm{Tpt}^{2-}$. Conformations of $\mathrm{Tpt}^{2-} 2 \mathrm{Me}^{+}$with the cations near one of the three outer rings of $\mathrm{Tpt}^{2-}$ could be ruled out since for these conformations a singlet ground state and a thermally-non-accessible triplet state is predicted, as was also the case for $\mathrm{Tpb}^{2-2} \mathrm{Me}^{+}[7]$, which is clearly in contrast with our experiments. Furthermore, the cations will prefer positions having the highest electron densities, which is for $\mathrm{Tpt}^{2-}$ on the nitrogen atoms. When considering both cation-cation repulsion and cation-anion polarization effects we found that the most favourable cation positions are above and below the same nitrogen atom.

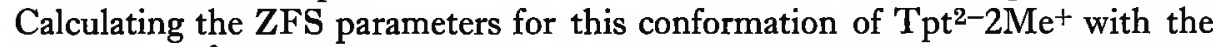
$\mathrm{Me}^{+}$ions at $2 \AA$ distance from the aromatic plane, we found (SCF $+\mathrm{CI}$ ) the following values: $D=440$ gauss; $E=40$ gauss. It is seen that the experimental $E$ value is well accounted for. It is, however, not justified to say that the above model is correct since the $D$ value deviates too much (i.e. 240 gauss) from the experimental value. Furthermore, the ground state is calculated to still be a triplet state, the energy gap between the triplet state and the lowest singlet state is $0.17 \mathrm{eV}$ $\left(1370 \mathrm{~cm}^{-1}\right)$.

Hence we must conclude that in contrast to the results obtained for triphenylene ${ }^{2-}[4]$ and $\mathrm{Tpb}^{2-}[7]$ the agreement between theory and experiment for $\mathrm{T} \mathrm{t}^{2-}$ and $\mathrm{Ttt}^{2-}$ is poor. To check whether the discrepancy would be solely due to the presence of the hetero atoms in these dianions, the ZFS parameter $D$ of the ultra-violet excited triplet state of Tpt was calculated following the same procedure as used for the dianion. The correspondence between the $D$ value obtained with the SCF + CI calculations and the experimental $D$ value of the Tpt triplet state was remarkably good

$$
\begin{aligned}
D(\text { calculated }) & =1270 \text { gauss, } \\
D(\text { experimental }) & =1320 \text { gauss [21]. }
\end{aligned}
$$

Further confirmation for the reliability of the method and of the used parameter values has been provided recently by De Jong and MacLean $[22,23]$ in calculating the ZFS parameters for several nitrogen containing aromatic compounds, using the 
same method and the same values for the semi-empirical parameters, except for $\beta_{\mathrm{CN}}$ which was taken equal to $-2 \cdot 74 \mathrm{eV}$ to fit the experimental $D$ value of the optical excited triplet state of quinoxaline. It is believed, therefore, that the theory is essentially reliable and that the discrepancy with experiment has another origin. As a possible explanation we suggest that the cations will force the phenyl ring(s) out of plane. This would certainly lower the singlet state with respect to the triplet state and would also increase the $D$ value.

The experimental assistance of Dr. W. P. J. Philipsen and Mr. H. L. Coenen is gratefully acknowledged.

\section{REFERENCES}

[1] Jesse, R. E., Biloen, P., Prins, R., van Voorst, J. D. W., and Hoijtink, G. J., 1963, Molec. Phys., 6, 633.

[2] van Willigen, H., Van Brokkhoven, J. A. M., and De Boer, E., 1967, Molec. Phys., $12,533$.

[3] Glasbeek, M., Visser, A. J. W., Maas, G. A., van Voorst, J. D. W., and Hoijtink, G. J., 1968, Chem. Phys. Lett., 2, 312.

[4] SommerdiJk, J. L., and De Boer, E., 1969, F. chem. Phys., 50, 4771.

[5] van Broekhoven, J. A. M., van Willigen, H., and De Boer, E., 1968, Molec. Phys., 15, 101.

[6] Glasbeek, M., 1969, Thesis, Amsterdam.

[7] Sommerdijk, J. L., van Broekroven, J. A. M., van Willigen, H., and De Boer, E., 1969, F. chem. Phys., 51, 2006.

[8] vaN BRoEkHoven, J. A. M., 1970, Thesis, Nijmegen.

[9] Murrell, J. N., and Hinchliffe, A., 1966, Molec. Phys., 11, 101.

[10] Shatenshtein, A. I. S., and Petrov, E. S., 1967, Russian chem. Revs, 36, 100.

[11] van Vleck, J. H., 1932, Electric and Magnetic Susceptibilities (Oxford University Press).

[12] STREITWIESER, A., 1967, Molecular Orbital Theory for Organic Chemists (John Wiley \& Sons).

[13] Goeppert Mayer, M., and SkLar, A. L., 1938, F. chem. Phys., 6, 645.

[14] Anno, T., and Sado, A., 1958, $\mathscr{F}$. chem. Phys., 29, 1168.

[15] Pariser, R., and Parr, R. G., 1953, F. chem. Phys., 21, 466, 767.

[16] Pariser, R., 1956, \%. chem. Phys., 24, 250.

[17] van Der Waals, J. H., and TER Maten, G., 1964, Molec. Phys., 8, 301.

[18] Capello, D., and Pullman, A., 1967, Theor. chim. Acta, 8, 383.

[19] McClelland, B. J., 1961, Trans. Faraday Soc., 57, 1458.

[20] REDdoch, A. H., 1967, Actes Colloq. Intern. No. 164, 'Structure Hyperfine Magnetique Atomes Molecules ', 419.

[21] Brinen, J. S., Koren, J. G., and Hodgson, W. G., 1966, f. chem. Phys., 44, 3095.

[22] De Jong, J., and MacLean, C., 1970, Chem. Phys. Lett, 5, 424.

[23] DE Jong, J., 1970, Thesis, V.U. Amsterdam.

[24] Pijpers, F. W., van Willigen, H., and Gerding, J. J. Th., 1967, Rec. Trav. chim. Pays-Bas Belg., 86, 511. 